\title{
Expectations of assisted conception for infertility
}

\author{
M G R Hull, H A Eddowes, U Fahy, M I Abuzeid, M S Mills, D J Cahill; C F Fleming, P G Wardle, \\ W C L Ford, A McDermott
}

Abstract

Objective-To provide reliable prognostic information for couples seeking assisted conception.

Design-Analysis of four years' practice (1988-91).

Setting-Private university service linked with NHS reproductive medicine services.

Patients -804 couples with various causes of subfertility, median duration five years, median age of women 34 years.

Interventions - 1280 completed cycles: 950 in vitro fertilisation, 144 gamete intrafallopian transfer, and 186 intrauterine insemination and superovulation.

Main outcome measures-Pregnancy and birth rates per cycle and cumulative pregnancy and take home baby rates per couple.

Results - In women under $\mathbf{4 0}$ years and men with normal sperm, whatever the cause of infertility, results with in vitro fertilisation improved steadily reaching a pregnancy rate per cycle of $30 \%(95 \%$ confidence interval $26 \%$ to $35 \%$ ) during $1990-1$ and birth rate per cycle of $29 \%$ (23\% to $35 \%)$ in 1990 . Pregnancy and birth rates for gamete intrafallopian transfer were $36 \%(28 \%$ to $44 \%)$ and $26 \%(17 \%$ to $37 \%)$ and for intrauterine insemination $18 \%(12 \%$ to $24 \%)$ and $16 \%$ (10\% to $22 \%)$. After six cycles cumulative probability of pregnancy was $82 \%$ and cumulative take home baby rate $70 \%$. Considering only in vitro fertilisation and gamete intrafallopian transfer after four cycles the pregnancy rate was $78 \%(66 \%$ to 91\%).

Conclusions-Conception is less likely in women over $\mathbf{4 0}$ and men with sperm dysfunction. For other couples the prognosis for a live birth is at least as good as for fertile couples if they persist with treatment.

\section{Introduction}

It has been difficult to provide infertile couples with a reliable prognosis for in vitro fertilisation and related methods of assisted conception because of selective reporting, varying criteria, including those for pregnancy (biochemical or clinical), and lack of birth data. In addition, there are now constraints on the number of embryos or eggs transferred per treatment. The World Health Organisation has also criticised and questioned the treatments, although it was as concerned by the financial cost to governments as by the cost in effort, stress, and risk to individuals.

This report aims at providing a reliable reference for prognostication by giving a complete account of recent practice (during the four years 1988-91) through to the completion of all pregnancies resulting from treatments up to the end of April 1991 (that is, births up to the end of January 1992). The numbers of eggs or embryos transferred per treatment has always been constrained by regulation to three, or in exceptional circumstances four, but since mid-1991 the absolute limit by law has been three.

Accurate prognostication for a couple must take account of the cause of infertility and the woman's age. Couples may choose treatment despite a poor chance of succeeding; often the main reason is to determine the fertilising ability of the spermatozoa when basic tests suggest sperm dysfunction and to help them come to terms with the diagnosis and turn, for example, to donor insemination instead. Reports of overall success rates can therefore be misleading. The results in this report are classified after analysis by the key factors found to affect chances of success in order to provide couples with useful therapeutic prognostication.

\section{Patients and methods}

Assisted conception methods are those which bring together specially prepared spermatozoa and eggs. The most common methods are in vitro fertilisation (IVF) with subsequent embryo transfer to the uterus, gamete intrafallopian transfer (GIFT), and high intrauterine insemination (IUI). In intrauterine insemination the fallopian tubes are flooded with spermatozoa at the time of ovulation but the technique is usually combined with superovulation as the chances of conception are further increased with multiple eggs. Our general philosophy is to start with in vitro fertilisation to provide diagnostic information about fertilising ability before considering the other treatments, if necessary.

Numerous other methods have been described, but so far their benefit is unconvincing except to overcome specific technical difficulties. We have recorded with the results of in vitro fertilisation the rare instances when cervical passage was impossible necessitating embryo transfer to the fallopian tubes or surgical needling of the uterine wall.

Couples were offered treatment according to the indications discussed in detail elsewhere. ${ }^{2}$ The main indications were tubal damage (unsuitable for surgery or failure to conceive after surgery); unexplained infertility for more than three years (apparently normal menstrual and ovulation cycles, normal spermcervical mucus interaction, and normal laparoscopic findings); endometriosis for more than two years; and sperm dysfunction. Our definition of male infertility was based not on standard seminal sperm counts but on tests of sperm function by penetration of normal cervical mucus or sperm migration into and motility in culture medium, or both. ${ }^{2}$ Men with low seminal sperm counts (oligospermia) but normal sperm function are therefore treated as having normal sperm. We excluded women only with ovarian failure or serious abnormality of the uterus. We made no restriction on sperm quality (provided that there was some function) or on the woman's age, but advised couples of the diminished chance of success in these cases and of the increased risks from pregnancy related and Gynaecology,

Bristol BS2 8EG

BMF 1992;304:1465-9 
to age. We may undertake relatively early treatment in women who are already approaching 40 years of age.

\section{METHODS OF ASSISTED CONCEPTION}

We used standard methods based on previously described techniques for in vitro fertilisation. ${ }^{3}$ Superovulation for in vitro fertilisation and gamete intrafallopian transfer was initially indicated with clomiphene and relatively low dose gonadotrophin, but during the past two and a half years we have used preliminary suppression of pituitary gonadotrophin secretion (pituitary down regulation) with buserelin, a gonadotrophin releasing hormone analogue, starting at the mid-luteal phase of the preceding ovarian cycle. Down regulation prevents a premature surge of luteinising hormone and so facilitates scheduling of egg collection; it also enables suppression of raised basal concentrations of luteinising hormones, which impair fertilisation and pregnancy rates and increase the risk of miscarriage.

After 10-14 days of buserelin treatment (by nasal spray) ovarian stimulation was begun by intramuscular injection of purified follicle stimulating hormone (urofollitrophin) two ampoules daily (150 units) to be increased after seven days if necessary. Ovarian follicular development was monitored by vaginal ultrasonography and measuring serum oestradiol concentrations. When ready for ovulation, the woman was given 5000 units of human chorionic gonadotrophin, and egg recovery was undertaken 35-36 hours later (about two hours before expected follicle rupture), usually by the vaginal ultrasound guided technique. Luteal function needed to be supported (because of suppressed endogenous luteinising hormone secretion) with further injections of human chorionic gonadotrophin (2000 units two and seven days after egg recovery), but progesterone supplementation was not routinely used. Embryos were transferred to the uterus through the cervix two days, sometimes three days, after egg recovery.

Sperm was usually prepared for in vitro fertilisation, gamete intrafallopian transfer, or intrauterine insemination by the swim-up technique, in which spermatozoa actively migrate into a covering layer of culture medium. In cases of sperm dysfunction preparation was optimised by appropriate modifications. These latterly included separation of the most favourable spermatozoa by passing them through a column of graduated densities of coated silicon particles (Percoll Pharmacia, Uppsala, Sweden).

Gamete intrafallopian transfer was undertaken only if the fallopian tubes were healthy, though tubes affected by adhesions due to endometriosis were acceptable if accessible. Preparation was as for in vitro fertilisation. Up to three eggs, or exceptionally four, were transferred by laparoscopy. Stimulation for intrauterine insemination was limited to a simple regimen of clomiphene followed by alternate day low dose follicle stimulating hormone (total usually six ampoules) and human chorionic gonadotrophin 5000 units. Intrauterine insemination was performed once 40 hours after giving human chorionic gonadotrophin only if the follicles had ovulated. All the recovered spermatozoa were injected in $0 \cdot 25-0.4 \mathrm{ml}$ of medium high into the uterus. Intrauterine insemination was usually cancelled if there were more than three mature follicles, though in vitro fertilisation or gamete intrafallopian transfer was offered instead.

\section{STATISTICAL ANALYSIS}

Results are expressed principally as pregnancy and birth rates per cycle of treatment reaching attempted egg recovery or intrauterine insemination, and compared with the $\chi^{2}$ test. Pregnancy was confirmed if there was ultrasonic evidence of a pregnancy sac (clinical pregnancy), not on the basis of earlier testing for human chorionic gonadotrophin (biochemical). Multiple births were counted as one; therefore more babies were born than indicated by the birth rates. Cumulative probabilities of pregnancy and birth per couple in successive cycles were calculated by the life table method, which accounts for couples not continuing as long as others in the study. Cumulative pregnancy rates refer to the first pregnancy for any couple. Cumulative birth rates refer to the first delivery of a surviving baby and include cycles of treatment leading up to any earlier pregnancy which might have miscarried. Standard errors for the cumulative rates were calculated by the Kaplan-Meier method, which takes account of the reduced number of couples proceeding to successive cycles of treatment. ${ }^{4}$ For comparison cumulative rates have been plotted with the best published rates in populations of proved fertility $^{56}$ and with birth rates (not excluding perinatal deaths) from Vessey et al. ${ }^{7}$

\section{Results}

During 1 January 1988 to 31 December 1991 attempted egg recovery or intrauterine insemination was reached in 1280 cycles in 804 couples; 950 in vitro fertilisation cycles, 144 gamete intrafallopian transfer, and 186 superovulation and intrauterine insemination. Other cycles were discontinued because of inadequate ovarian response (mainly in women over 40 years old: $21 \%$ of their cycles) or premature ovulation when pituitary down regulation had not been used (18\% of cycles in women under 40 years old). Only $5 \%$ of down regulated cycles in women under 40 years old were discontinued because of inadequate or, less often, excessive ovarian response. One patient conceived naturally after stimulation alone because the ovarian response was considered inadequate to proceed to in vitro fertilisation.

The median age of the women was 34 (range 22-46) years; 723 were aged less than 40 years (median 32 years) and 91 aged 40 or more (median 41 years) when treated; 10 were treated both before and after reaching 40 years. The median duration of infertility in all women of all ages was five (ringes 1-20) years and 330 $(41 \%)$ women had secondary infertility. The main diagnosis was tubal disease in 289 , endometriosis in 143 , sperm dysfunction in 117 , and unexplained infertility in 159. Another 76 had largely unexplained infertility, although reliable sperm mucus testing results were not available, and the remaining 20 had miscellaneous minor abnormalities such as cervical mucus defects, fibroids, and endometrial adhesions of uncertain relevance. The distributions of diagnoses were significantly different in women with primary and secondary infertility $(p<0.001)$ because tubal disease was more common in secondary infertility ( 186 women, $56 \%)$ than primary infertility $(103,22 \%)$

Preliminary analysis of the results showed no difference in successful pregnancy rates between women with primary and secondary infertility, or between women aged less than 35 and 35-39 years old. There was significant but only slight reduction in fertilisation rates per egg (given normal sperm) in women with endometriosis ( $52 \%)$, and unexplained infertility (59\%) compared with tubal disease $(66 \% ; p<0 \cdot 01)$, but there were usually sufficient eggs to achieve three embryos for transfer and pregnancy rates by in vitro fertilisation were unaffected. By contrast, fertilisation rates were substantially reduced in cases of sperm dysfunction $(41 / 124,33 \%, p<0.001)$ and pregnancy rates halved. The following analyses have therefore been classified according to the woman's age ( $<40 v \geqslant 40$ years) and previous diagnosis of sperm dysfunction.

Table I summarises the results of in vitro fertilisation 


\begin{tabular}{|c|c|c|c|c|c|c|c|c|c|c|c|}
\hline \multirow[b]{2}{*}{ Age and diagnosis } & \multicolumn{3}{|c|}{1988} & \multicolumn{3}{|c|}{1989} & \multicolumn{3}{|c|}{1990} & \multicolumn{2}{|c|}{1991} \\
\hline & $\begin{array}{l}\text { No of } \\
\text { cycles }\end{array}$ & $\begin{array}{c}\text { No }(\%) \text { of } \\
\text { pregnancies }\end{array}$ & $\begin{array}{c}\text { No (\%) of } \\
\text { births }\end{array}$ & $\begin{array}{l}\text { No of } \\
\text { cycles }\end{array}$ & $\begin{array}{c}\text { No }(\%) \text { of } \\
\text { pregnancies }\end{array}$ & $\begin{array}{l}\text { No (\%) of } \\
\text { births }\end{array}$ & $\begin{array}{l}\text { No of } \\
\text { cycles }\end{array}$ & $\begin{array}{c}\text { No (\%) of } \\
\text { pregnancies }\end{array}$ & $\begin{array}{c}\text { No (\%) of } \\
\text { births }\end{array}$ & $\begin{array}{l}\text { No of } \\
\text { cycles }\end{array}$ & $\begin{array}{c}\text { No (\%) of } \\
\text { pregnancies }\end{array}$ \\
\hline \multicolumn{12}{|l|}{ Women aged $\geqslant 40$ : } \\
\hline Sperm dysfunction & 3 & & & 4 & & & 3 & & & 2 & \\
\hline Tubal disease & 9 & $3(33)$ & $2(22)$ & 4 & & & 8 & $1(13)$ & $1(13)$ & 14 & $2(14)$ \\
\hline Others & 10 & $1(10)$ & & 11 & $1(9 \%)$ & & 11 & $3(27)$ & $1(9)$ & 12 & $1(8)$ \\
\hline \multicolumn{12}{|l|}{ Women $<40$ : } \\
\hline Sperm dysfunction & 22 & & & 31 & $1(3)$ & & 35 & $7(20)$ & $7(20)$ & 41 & $6(15)$ \\
\hline Tubal disease & 82 & $21(26)$ & $14(17)$ & 84 & $14(17)$ & $13(15)$ & 107 & $33(31)$ & $29(27)$ & 115 & $31(27)$ \\
\hline Others & 52 & $4(8)$ & $4(8)$ & 88 & $23(26)$ & $21(24)$ & 95 & $30(32)$ & $30(32)$ & 107 & $35(33)$ \\
\hline All women & 178 & $28(16)$ & $20(11)$ & 222 & $39(18)$ & $34(15)$ & 259 & $74(29)$ & $68(26)$ & 291 & $75(26)$ \\
\hline
\end{tabular}

TABLE II-Results of gamete intrafallopian transfer or superovulation and intrauterine insemination by woman's age and year of treatment. Treatments were never undertaken in women with tubal disease, and usually only after previously shown fertilisation in vitro, so cases of sperm dysfunction are not distinguished

\begin{tabular}{|c|c|c|c|c|c|c|}
\hline \multirow[b]{2}{*}{ Treatment age (years) } & \multicolumn{3}{|c|}{ 1988-91 } & \multicolumn{3}{|c|}{$1988-90$} \\
\hline & $\begin{array}{l}\text { No of } \\
\text { cycles }\end{array}$ & $\begin{array}{c}\mathrm{No}(\%) \text { of } \\
\text { pregnancies }\end{array}$ & $\begin{array}{c}95 \% \\
\text { Confidence } \\
\text { interval (\%) }\end{array}$ & $\begin{array}{l}\text { No of } \\
\text { cycles }\end{array}$ & $\begin{array}{l}\text { No }(\%) \text { of } \\
\text { births }\end{array}$ & $\begin{array}{c}95 \% \\
\text { Confidence } \\
\text { intervals (\%) }\end{array}$ \\
\hline \multicolumn{7}{|c|}{ Gamete intrafallopian transfer: } \\
\hline Woman $\geqslant 40$ & 20 & $2(10)$ & 1 to 32 & 17 & $1(6)$ & 0.2 to 29 \\
\hline \multirow{2}{*}{\multicolumn{7}{|c|}{$\begin{array}{l}\text { Superovulation and intrauterine } \\
\text { insemination: }\end{array}$}} \\
\hline & & & & & & \\
\hline Woman $\geqslant 40$ & 28 & $1(4)$ & $0 \cdot 1$ to 18 & 28 & $1(4)$ & $0 \cdot 1$ to 18 \\
\hline Woman $<40$ & 158 & $29(18)$ & 12 to 24 & 152 & $24(16)$ & 10 to 22 \\
\hline
\end{tabular}

in terms of pregnancy and birth rates per cycle. Women with tubal disease are distinguished from those with other causes of infertility because those with other causes are also suitable for gamete intrafallopian transfer or intrauterine insemination. The results of these treatments are given in table II. Pregnancy and birth rates after in vitro fertilisation increased significantly $(\mathrm{p}<0.01)$ in the second year (1989) and third year (1990), both overall and in each diagnostic subgroup in women under 40 years old (table I).

The overall pregnancy rates per cycle of in vitro fertilisation, in all ages and diagnostic groups, for the years $1988-91$ were $16 \%, 18 \%, 29 \%$, and $26 \%$ and the birth rates for $1988-90$ were $11 \%, 15 \%$, and $26 \%$. In women under 40 years old and men with normal sperm (combining the tubal disease and other categories) the pregnancy rates in successive years were $19 \%, 22 \%$, $31 \%$, and $30 \%$ and the birth rates $13 \%, 20 \%$, and $29 \%$. In the same group the overall pregnancy rate per cycle in the four years $1988-91$ was $26 \%$ (95\% confidence intervals $23 \%$ to $29 \%$ ) and the birth rate in the three years $1988-90$ was $22 \%$ (18\% to $25 \%$ ). Reflecting current improved practice, the pregnancy rate per cycle in $1990-1$ was $30 \%(26 \%$ to $35 \%)$ and birth rate from treatments in 1990 was $29 \%$ ( $23 \%$ to $35 \%$ ).

The average implantation rate per individual embryo transferred in women aged under 40 in $1988-91$ was $14 \%$ when up to three embryos were transferred but only $7 \%(\mathrm{p}<0.01)$ when four embryos were transferred (reflecting the adverse indications for four embryo transfers). The implantation rate for up to three embryos was, however, significantly higher in $1990-1(15 \%)$ than in $1988-9(11 \% ; \mathrm{p}<0.025)$.

Table II gives the overall pregnancy and birth rates for gamete intrafallopian transfer and intrauterine insemination. Different diagnoses are not distinguished because no women had tubal disease and the couples with sperm dysfunction treated by gamete intrafallopian transfer or intrauterine insemination almost invariably had had their fertilising ability proved by in vitro fertilisation. The implantation rate per individual oocyte transferred by gamete intrafallopian transfer in women aged under 40 was $18 \%$.

The women who conceived were of similar age (median 34, range 22-45 years) and duration of infer- tility (median 5, 1-19 years) as those who failed to conceive. The outcome is known for 1036 treatment cycles up to the end of April 1991 (births up to end of January 1992). The rate of pregnancy miscarriage was $45 \%(5 / 11)$ in women over 40 and $8.5 \%(18 / 212)$ in those under 40; ectopic pregnancy occurred in $12 / 212$ $(5 \cdot 7 \%)$ women under 40 (and none over 40 ) including one successful heterotopic (intrauterine and extrauterine) pregnancy. Multiple births occurred only in women under 40 ; the rate was $27 \%$ : 42 twins $(23 \%)$ and eight triplets (4\%). Perinatal mortality was $4 \cdot 0 \%$, due mainly to prematurity associated with multiple pregnancy, but only $3 / 189(1.6 \%)$ women who gave birth had no surviving child and in two out of the three cases there was a stillborn singleton.

Figures 1 and 2 show the cumulative pregnancy and successful birth (take home baby) rates per couple for each type of treatment and for all treatments received in women under 40 years old and men with normal sperm, as these were the only couples encouraged to persist with treatment. Gamete intrafallopian transfer and intrauterine insemination were nearly always undertaken after previous in vitro fertilisation. Conception rates did not seem to reduce in successive cycles. All births up to the end of January 1992 from treatments up to the end of April 1991 are included. The cumulative birth rates cover more cycles of treatment than the pregnancy rates because some births resulted from a second pregnancy after earlier treatment had led to a miscarriage. The graphs have not been extended to their limits because of the distorting effect of small numbers. For example, not shown on the graphs for "all methods" is that one couple continued to a seventh cycle of treatment and conceived (for the first time) and one couple continued to a 10th cycle of treatment, having miscarried on the way, and had a live baby-if included, these results would suggest that $100 \%$ success is achievable.

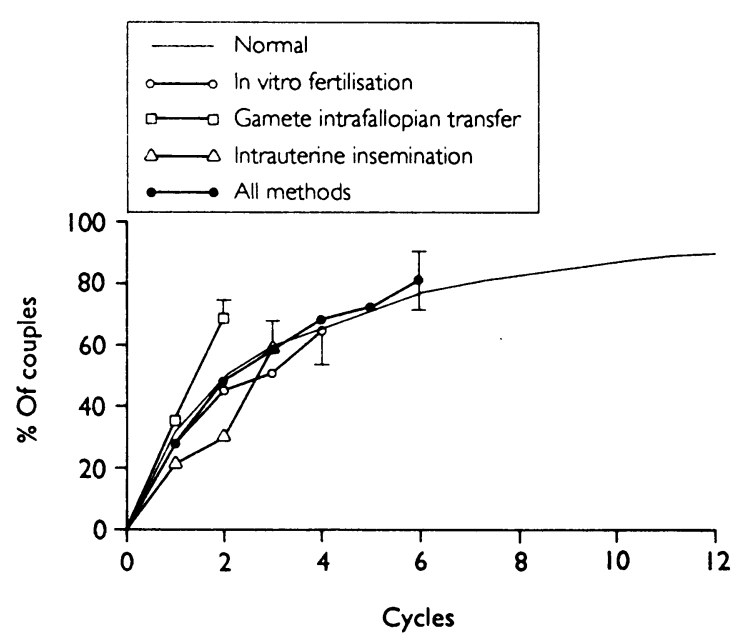

FIG 1-Cumulative pregnancy rates by assisted conception methods individually or in serial combinations in women under 40 years old and men with normal sperm, compared with a normal population of proved fertility. ${ }^{5}$ Bars show standard error 


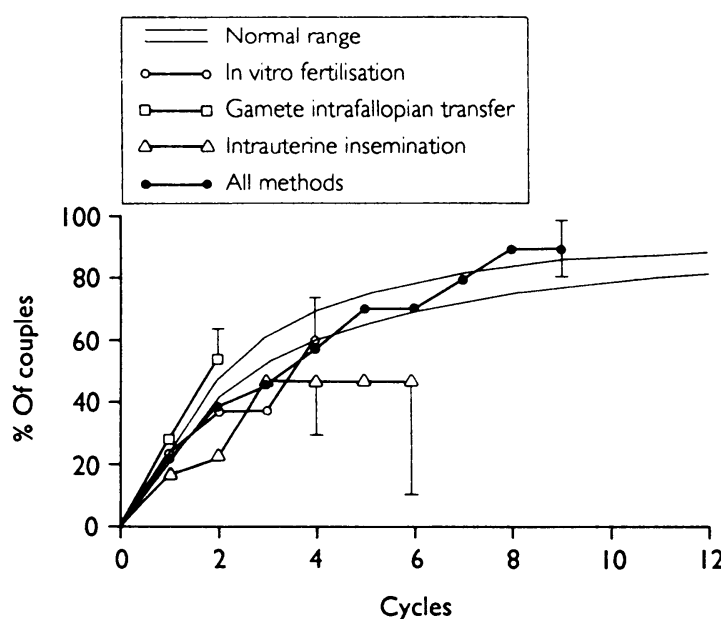

FIG 2-Cumulative successful birth rates in women under 40 years old and men with normal sperm. Some first pregnancies miscarried. Normal reference population is shown by two curves, the upper for parous women and the lower nulligravid women (uncorrected for perinatal mortality $)^{7}$

Combining the results of in vitro fertilisation and gamete intrafallopian transfer the cumulative probability of pregnancy after four cycles was $78 \%$ (66\% to $91 \%)$. The take home baby rate after four cycles seemed higher $(83 \%, 71 \%$ to $94 \%)$ due to the distorting effect of a small group in the final cycle, the overall group being smaller because the latest pregnancies have not been completed.

\section{Discussion}

We have described the complete results of an assisted conception unit over the past four years. Our results therefore provide an accurate and reliable indication of the chance of success for individual couples treated in such a unit under current conditions, with respect to both the methods of treatment used and the current regulatory constraints of the United Kingdom's licensing authority (the Human Fertilisation and Embryology Authority). In particular the numbers of embryos or eggs transferred by in vitro fertilisation or gamete intrafallopian transfer is now restricted to three in each case. Before the new legislation four embryos or eggs were transferred only in exceptional adverse circumstances, in our unit, and this was reflected in reduced implantation and pregnancy rates. The circumstances for four embryo transfers included, for instance, women over 40 years old, or apparently poorly developing embryos.

Our findings show that for both scientific purpose and clinical prognostication results must be classified by certain key conditions. In practice, this can be done simply by distinguishing women over 40 years old and men with dysfunctional sperm. Of course women with ovarian failure or serious uterine abnormality are not suitable for such treatment. We found that the woman's age (except above 40 years), no previous pregnancy, or length of infertility did not affect the chances of successful pregnancy. Thus while women over 40 years and men with sperm dysfunction must be advised about their reduced chance of success, which is compounded in women over 40 years by increased risk of miscarriage, the remaining majority of couples can be given a common prognosis qualified only by whether they are suitable for gamete intrafallopian transfer or superovulation and intrauterine insemination. In vitro fertilisation should always be used for women with damaged fallopian tubes even if patent.

In women under 40 years and men with normal sperm, whatever the cause of infertility, clinical pregnancy rates per cycle by in vitro fertilisation, gamete intrafallopian transfer, and superovulation and intra- uterine insemination averaged $26 \%, 36 \%$, and $18 \%$ respectively and birth rates were $22 \%, 26 \%$, and $16 \%$. There was a significant improvement in results with in vitro fertilisation, however, in the second and third years, and current expectation of success by in vitro fertilisation would be closer to the average pregnancy rate during $1990-1$ of $30 \%$ and the average birth rate from treatments in 1990 (the last treatment year of completed pregnancies) of $29 \%$.

Figures 1 and 2 show the cumulative probabilities of a couple (woman under 40, man with normal sperm) achieving pregnancy and having a baby. They show that with any combination of appropriately selected treatment-commonly in vitro fertilisation followed by gamete intrafallopian transfer or superovulation and intrauterine insemination $-82 \%$ of couples prepared to continue for six cycles can be expected to conceive and $70 \%$ to have a surviving baby. Some who conceive miscarry, though the risk is no greater than after natural conception when pregnancy has been shown by ultrasonography. If couples are prepared to have further treatment it seems that after nine cycles of treatment $90 \%$ can expect to have a baby or at least $72 \%$ as indicated by the lower $95 \%$ confidence limit. With only in vitro fertilisation or gamete intrafallopian transfer, however, the expectation of having a baby is at least $71 \%$ after only four cycles.

The cumulative success rates can be seen to be at least as good as for natural conception in fertile couples. Comparison with natural conception is not strictly accurate, however, because treatment is unlikely to be offered in successive months. Nevertheless, the rates with treatment are impressively higher than the natural chance of pregnancy in those infertile (or subfertile) couples, which is around $0-20 \%$ after 12 months or $0-30 \%$ after two years. ${ }^{2}$

There seems to be no limit to the number of cycles of treatment which couples without unfavourable indications should be encouraged to pursue because their chance of success is maintained in successive cycles, at least as far as our experience has gone so far. Others have found the same, ${ }^{8-12}$ The main limiting factor is the emotional, physical, and financial cost, and most couples seem to feel satisfied that they have tried hard enough after two to three cycles.

Figure 3 compares our cumulative results with those in other reports..$^{8-12}$ Though rates vary, all the reports show that the chance of pregnancy is maintained in successive cycles. Some go back to treatment several years ago and do not have the advantage of what seems to have been a widely experienced improvement in recent years. For example, a European multicentre

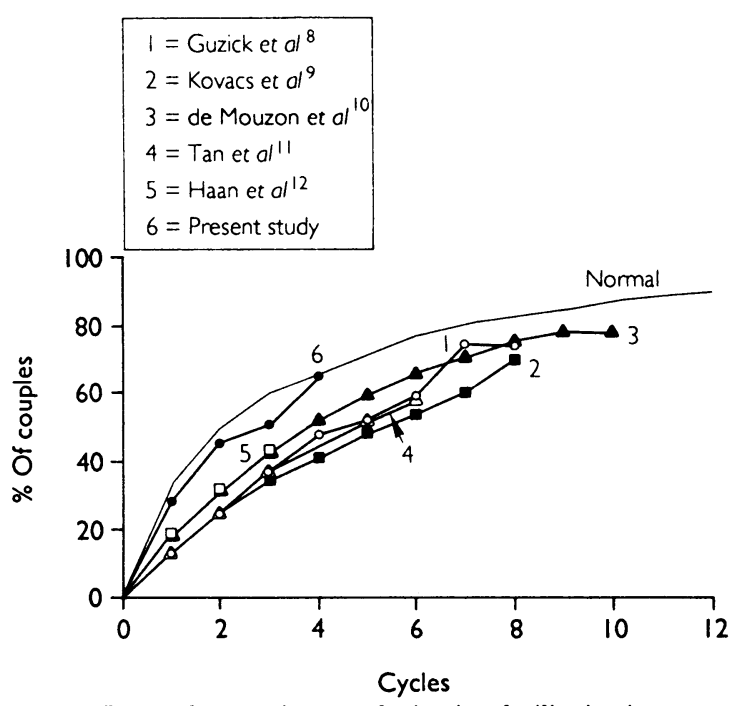

FIG 3-Reported conception rates by in vitro fertilisation in women under 35-40 years old 
study of treatment during $1989-90$ for prolonged unexplained infertility found average pregnancy rates per cycle for in vitro fertilisation, gamete intrafallopian transfer, and superovulation and intrauterine insemination of $28 \%, 29 \%$, and $23 \%$ respectively. ${ }^{13}$ Unfortunately these results cannot be strictly compared with ours because there was no information about the numbers of embryos or eggs transferred, or of follicles stimulated for intrauterine insemination. The chance of pregnancy with intrauterine insemination is likely to be directly related to the number of follicles stimulated but this also increases the risk of high order multiple pregnancy. Cumulative pregnancy rates by superovulation and intrauterine insemination in couples with appropriate indications have been reported to reach $56 \%{ }^{14}$ and $68 \%{ }^{15}$ after six cycles of treatment, compared with our finding of $59 \%$ after four cycles. There are no useful published cumulative data for gamete intrafallopian transfer.

A strict comparison of in vitro fertilisation, gamete intrafallopian transfer, and superovulation and intrauterine insemination in our unit during 1988-90 showed significant benefit from gamete intrafallopian transfer (in appropriate cases), with respective pregnancy rates per cycle of $28 \%, 40 \%$, and $20 \% .{ }^{16}$ Our improvements in in vitro fertilisation during the past two years (see tables I and II) seem, however, to have greatly narrowed the gap.

The reasons for the improvements specifically in in vitro fertilisation can only be speculated. Perhaps the technical learning process is longer, but we had earlier experience in a research programme and others have found the same. We also found a significant improvement in successful implantation of embryos, which suggests a beneficial change in local environmental factors. It is tempting to attribute the improvement to the routine use of gonadotrophin releasing hormone analogues which down regulate pituitary gonadotrophin secretion. It has certainly reduced the rate of cancellation of treatment substantially, and for that reason alone seems cost effective.

Another important advance has been in sperm preparation methods in cases of sperm disorder, using a Percoll density gradient column. ${ }^{17}$ The pregnancy rate per cycle of in vitro fertilisation during the past two years was $17 \%$ in cases of sperm dysfunction, which is encouraging enough to usually try in vitro fertilisation, at least initially to assess fertilising ability

In women over 40 years old the results have also been moderately encouraging, though it seems clear that only the most efficient treatments-namely in vitro fertilisation and gamete intrafallopian transfer-are worth trying, giving an average pregnancy rate per cycle of $14 \%$. However, nearly half the pregnancies miscarried. The reduced success in women over 40 years challenges the regulatory constraint on numbers of eggs or embryos transferred, but freedom from constraint would require willingness to reduce multiple pregnancies by destructive intervention.

In women under 40 years old superovulation and intrauterine insemination offer half the chance of success compared with gamete intrafallopian transfer in suitable cases but may be considered a cost effective choice in women not yet approaching 40 years.

We are grateful for the essential expert contribution of our team of laboratory scientists Barbara Ray, Jennifer Williams, Christopher Harlow, Shona Grant, and Lynn Nice; specialis nurses Josephine Mitchell, Katherine Franklin, Gillian Philip, Catherine Prosser, Janice Hayhurst, Amanda Rogers, Patricia Diamond, Carole Sleight, Susan Heyworth, Milanka Radojcic, and Elizabeth Corrigan; and gynaecologists Naomi Whitelaw and Robert Fox. We also thank Eileen McLaughlin for preparing the illustrations.

1 World Health Organisation. Consultation on the place of in vitro fertilization in infertility care. WHO: Geneva, 1990. Report EUR/ICP/MCH 122(S).

2 Hull MGR. Indications for assisted conception. Br Med Bull 1990;46:580-95. (Part 3. Edwards RG, ed. Assisted human conception.)

3 Wardle PG, Mitchell JD, McLaughlin EA, Ray BD, McDermott A, Hull MGR. Endometriosis and ovulatory disorder: reduced fertilisation in vitro compared with tubal and unexplained infertility. Lancet 1985 ;ii:236-9.

4 Matthews DE, Farewell VT. Using and understanding medical statistics. 2nd ed. Basle: Karger, 1988:75-8.

5 Tietze C. Statistical contributions to the study of human fertility. Fertil Steril 1956;7:88-95.

6 Tietze $C$. Fertility after the discontinuation of intrauterine and oral contraception. Int $\mathcal{F}$ Fertil 1986;13:385-9.

7 Vessey MP, Wright NH, McPherson K, Wiggins P. Fertility after stopping different methods of contraception. BMF 1978;i:265-7.

8 Guzick DS, Wilkes C, Jones HW. Cumulative pregnancy rates for in vitro fertilization. Fertil Steril 1986;46:663-7.

9 Kovacs GT, Rogers P, Leeton JF, Trounson AO, Wood C, Baker HWG. In vitro fertilisation and embryo transfer. Prospects of pregnancy by life table analysis. Med f Aust 1986;144:682-3.

10 de Mouzon J, Bachelot A, Gagnepain A, Pessione F. Analyse des resultats de FIVETE) 1989 et 1986-1989. Contraception-Fertilite-Sexualite 1990;18:589-91.

11 Tan SL, Steer C, Royston P, Rizk P, Mason BA, Campbell S. Conception rates and in vitro fertilisation. Lancet 1990:335:299.

12 Haan G, Bernardus RE, Hollanders HMG, Leerentveld BA, Prak FM, Naaktgeboren $N$. Selective drop-out in successive in-vitro fertilization attempts: the pendulum effect. Hum Reprod 1991;6:939-43.

13 Crosignani PG, Walters DE, Soliani A. The ESHRE multicentre trial on the treatment of unexplained infertility: a preliminary report. Hum Reprod 1991;6:953-8.

14 Dodson WC, Haney AF. Controlled ovarian hyperstimulation and intrauterine insemination for treatment of infertility. Fertil Steril 1991;55: 457-67.

15 Chaffkin LM, Nulsen JC, Luciano AA, Metzger DA. A comparative analysi of the cycle fecundity rates associated with combined human menopausa gonadotrophin (hMG) and intrauterine insemination (IUI) versus either hMG or IUI alone. Fertil Steril 1991;55:252-7.

16 Mills MS, Eddowes HA, Cahill DJ, Abuzeid MIM, McDermott A, et al. A controlled study of in-vitro fertilization (IVF), gamete intrafallopian transfer (GIFT) and intrauterine insemination (IUI) combined with superovulation. Hum Reprod (in press).

17 Nice L, Ray B, Grant S, Williams J, McDermott A, Hull MGR. Use of Percoll in IVF: a comparison between sperm dysfunction and tubal patients. I Reprod Fertil Abstr Ser 1991;7:48.

(Accepted 19 fanuary 1992)

\section{ONE HUNDRED YEARS AGO}

UNSATISFACTORY INQUESTS

It is much to be hoped that in the new Parliament someone will take up and carry into effect a very considerable reform in regard to coroners' inquests. Here are samples of the way inquests are at present conducted at Walsall. A child, aged 5, complained of pain in his stomach one Saturday, and was given medicine from a chemist's, after which he appeared relieved; next morning he was sick, became worse in the course of the day, and died suddenly in the afternoon. A neighbour who was present at the death testified that in her opinion it was a case of convulsions, and a verdict was accordingly returned to this effect. When an inquest is thus held, it is an empty farce, and it is perfectly scandalous that such worthless investigations should be possible. Not one tittle of evidence was produced to show whether the child's death was due to natural causes or not. An inquiry was recently held at Tunbridge on the body of an infant who had helped itself to a dose of about a drachm of a solution of chloral, and died some hours later. The death was clearly due to misadventure, and with that we do not concern ourselves but a medical witness - not the medical man from whom the chloral was obtained six and a-half years previously who was not called to the inquest at all-is reported to have said that 1 grain of chloral would kill a child (the deceased was 2 years old). The smallest fatal dose we know of is 3 grains, which killed a child of 1 year. The chlora had been prescribed for an older child for toothache, a line of treatment to which, notwithstanding the opinion of the practitioner referred to, we know of no valid objection. We gather that no post-mortem examination was made in this case; and, as tracheotomy had been performed, and as the death took place some hours after the poisoning, and when apparently all the narcotic symptoms had passed away, the exact cause could not be determined.

(BMF 1892;ii;:34) 